\title{
Saquinavir/zidovudine combination in patients with advanced HIV infection and no prior antiretroviral therapy: CD4 + lymphocyte/plasma RNA changes, and emergence of HIV strains with reduced phenotypic sensitivity
}

Stefano Vella ${ }^{\mathrm{a}}$, Clementina Galluzzo ${ }^{\mathrm{a}}$, Giacomo Gianninia ${ }^{\mathrm{a}}$, Maria F. Pirillo ${ }^{\mathrm{a}}$, Ian Duncan $^{\text {b }}$, Helmut Jacobsen ${ }^{\mathrm{c}}$, Massimo Andreoni ${ }^{\mathrm{d}}$, Loredana Sarmati ${ }^{\mathrm{d}}$, Lucia Ercoli ${ }^{\mathrm{d}}$

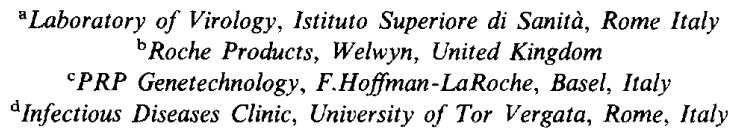

Keywords: HIV; Saquinavir; Zidovudine

A randomized, double-blind study was performed to assess the antiviral activity and tolerability of the HIV-proteinase inhibitor saquinavir (SQV), alone or in combination with zidovudine (ZDV).

A total of 92 previously untreated HIV-infected patients with CD4 + cell count of less than $300 /$ $\mathrm{mm}^{3}$ were randomized to receive one of five treatments, three times a day: SQV $600 \mathrm{mg}$, ZDV 200 $\mathrm{mg}$, SQV 75,200 or $600 \mathrm{mg}$ in combination with ZDV $200 \mathrm{mg}$. The primary treatment period was 16 weeks, with blinded monthly extensions [1].

The mean CD4 + changes over 24 week period (DAVG analysis) in the five arms are presented in Fig. 1. The median baseline $\mathrm{CD} 4+$ counts ranged from 156 to 248 cells $/ \mathrm{mm}^{3}$. In the SQV/ $\mathrm{ZDV}$ combination arm, the peak $\mathrm{CD}^{+}{ }^{+}$increase was about 60 cells $/ \mathrm{mm}^{3}$ at week 16 . The same DAVG analysis at 24 weeks with respect to reduc- tion in plasma viral load measured by quantitative HIV-RNA RT-PCR is presented in Fig. 2. The median baseline RNA copies ranged from 5.2 to $5.3 \log _{10} / \mathrm{ml}$. For the $600 \mathrm{mg}$ SQV plus ZDV combination arm, a peak decrease of $-1.6 \log _{10}$ was observed at week 2 .

No reduction in phenotypic sensitivity to either drug was detected in virus isolated at baseline. At week 16 , a reduced sensitivity to SQV (defined as $\mathrm{IC}_{50}>10 \mathrm{nM}$ ) was present in 3 out of 12 patients on SQV monotherapy (mean $\mathrm{IC}_{50}$ of the three patients: $23.3 \mathrm{nM}$; range: $11-39 \mathrm{nM}$ ), while no reduced sensitivity to SQV was detected among isolates obtained from 17 patients receiving SQV $600 \mathrm{mg}$ in combination with ZDV and from 14 patients receiving ZDV monotherapy (Table 1). No reduced sensitivity to ZDV was detected at week 16 in any of the 17 viral isolates from patients in the $600 \mathrm{mg}$ 


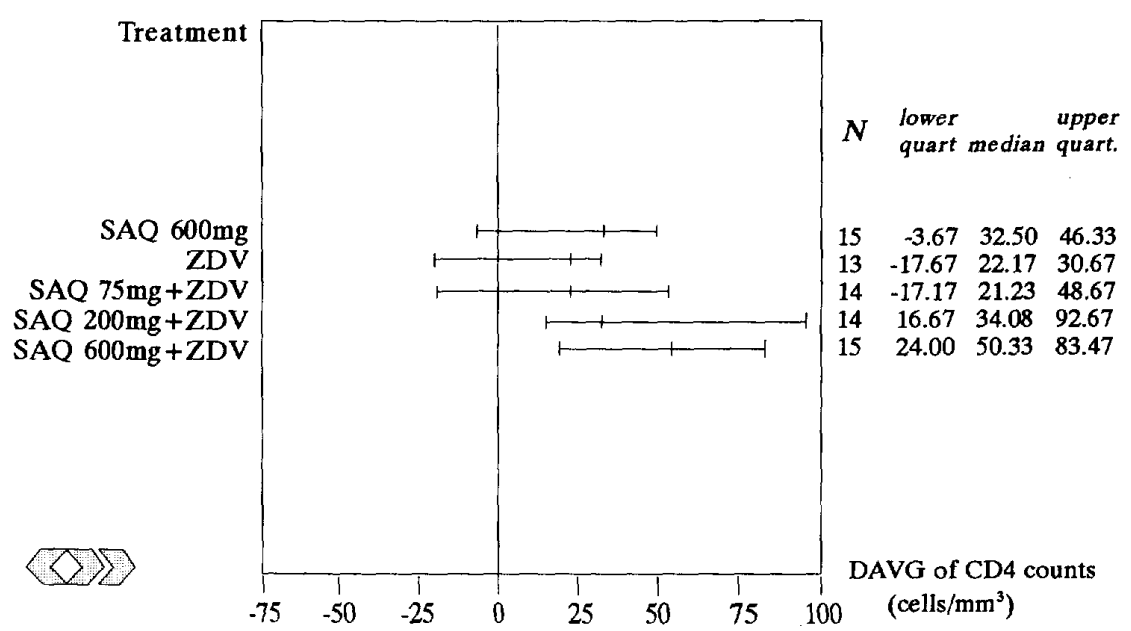

Fig. 1. ZDV/Saquinavir combination in naive PTS. Integrated comparison of CD4 counts. Change over a 24-week period (DAVG).

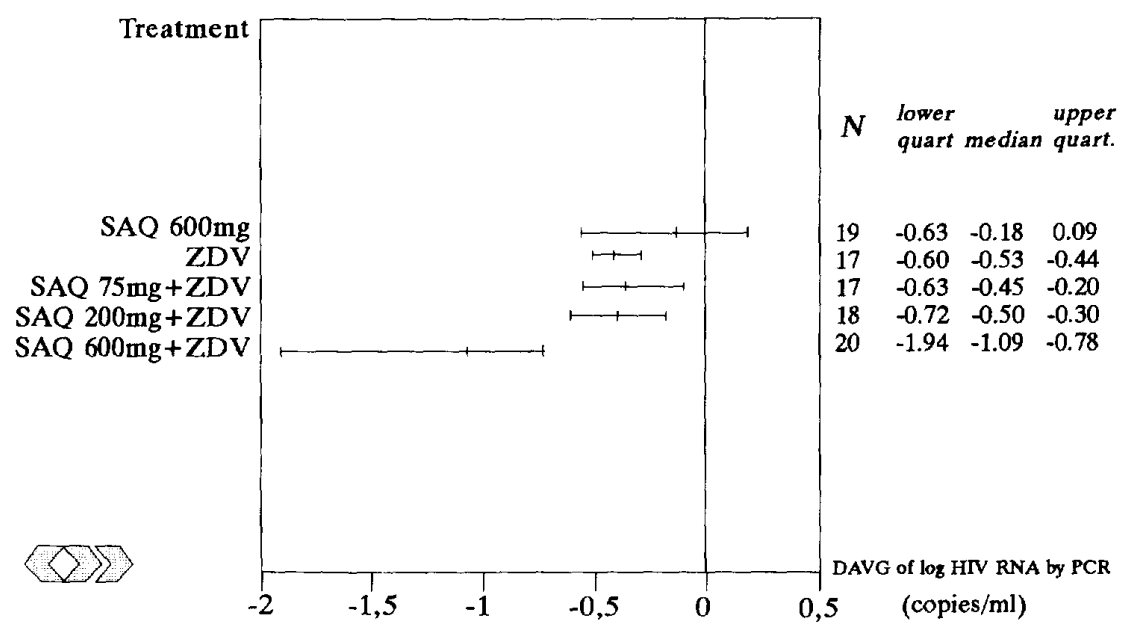

Fig. 2. AZT/Saquinavir combination in naive PTS. Integrated comparison of log HIV RNA copy by PCR. Change over a 24-week period (DAVG).

SQV plus ZDV combination arm, while reduced sensitivity to ZDV was detected in 3 out of 14 patients taking ZDV monotherapy (mean $\mathrm{IC}_{50}$ : $0.08 \mu \mathrm{M}$; range: $0.07-0.09 \mu \mathrm{M}$ ) (Table 2).

Drug sensitivity studies on HIV plasma strains isolated during the extension phase showed that, after 8-12 months, 7 out of 11 and 3 out of 13 patients, in the SQV and SQV/ZDV arms, respectively, harboured HIV strains with reduced sensitivity to SQV. With respect to ZDV, 10 out of 13 patients in the ZDV monotherapy arm had HIV isolates with reduced sensitivity (defined as viral 
Table 1

Reduced sensitivity to Saquinavir. Plasma isolates

\begin{tabular}{lll}
\hline Treatment arm (tid) & \multicolumn{2}{l}{$\begin{array}{l}\text { N. with reduced sensitivity } \\
\text { tested }\end{array}$} \\
\cline { 3 - 3 } & \multicolumn{2}{l}{ Time on therapy } \\
\cline { 2 - 3 } & 4 months & $8-12$ months \\
\hline ZDV 200 mg & $3 / 13$ & $7 / 11$ \\
SAQ 75 mg plus ZDV 200 mg & $0 / 05$ & - \\
SAQ 200 mg plus ZDV 200 mg & $0 / 4$ & - \\
SAQ 600 mg plus ZDV 200 mg & $0 / 17$ & $7 / 13$ \\
SAQ 600 mg & $0 / 13$ & $0 / 13$ \\
\hline
\end{tabular}

$\mathrm{IC}_{50}>10 \mathrm{nM}$.

a No patient received antiretroviral therapy before enrollment.

isolates with $\mathrm{IC}_{50}>0.045 \mu \mathrm{M}$ ), while only 2 out of 13 isolates from patients in the SQV/ZDV combination arm had reduced sensitivity to ZDV $(P<<0.001)$ (Tables 1 and 2$)$.

Overall, drug sensitivity results suggest that a
Table 2

Reduced sensitivity to Zidovudine. Plasma isolates

\begin{tabular}{lll}
\hline Treatment arm (tid) & \multicolumn{2}{l}{$\begin{array}{l}\text { N. with reduced sensitivity/ } \\
\text { tested }\end{array}$} \\
\cline { 2 - 3 } & \multicolumn{2}{l}{ Time on therapy } \\
\cline { 2 - 3 } & 4 months & $8-12$ months \\
& $3 / 14$ & $10 / 13^{*}$ \\
ZDV 200 mg & $2 / 5$ & - \\
SAQ 75 mg plus ZDV 200 mg & $0 / 4$ & - \\
SAQ 200 mg plus ZDV 200 mg & $0 / 4$ \\
SAQ 600 mg plus ZDV 200 mg & $0 / 17$ & $2 / 13^{*}$ \\
SAQ 600 mg & $0 / 13$ & $0 / 11$ \\
\hline
\end{tabular}

$\mathrm{IC}_{50}>0.045 \mathrm{nM}$

a No patient received antiretroviral therapy before enrollment. $* P<0.01$.

combination of SQV and ZDV may delay the emergence of phenotypic resistance to either drug in the short term, and to $\mathrm{ZDV}$ at least after one year. 